\title{
"||||||||||||||||||||||||||||||||||||||||||||||||||||||||||||||||||||||.
}

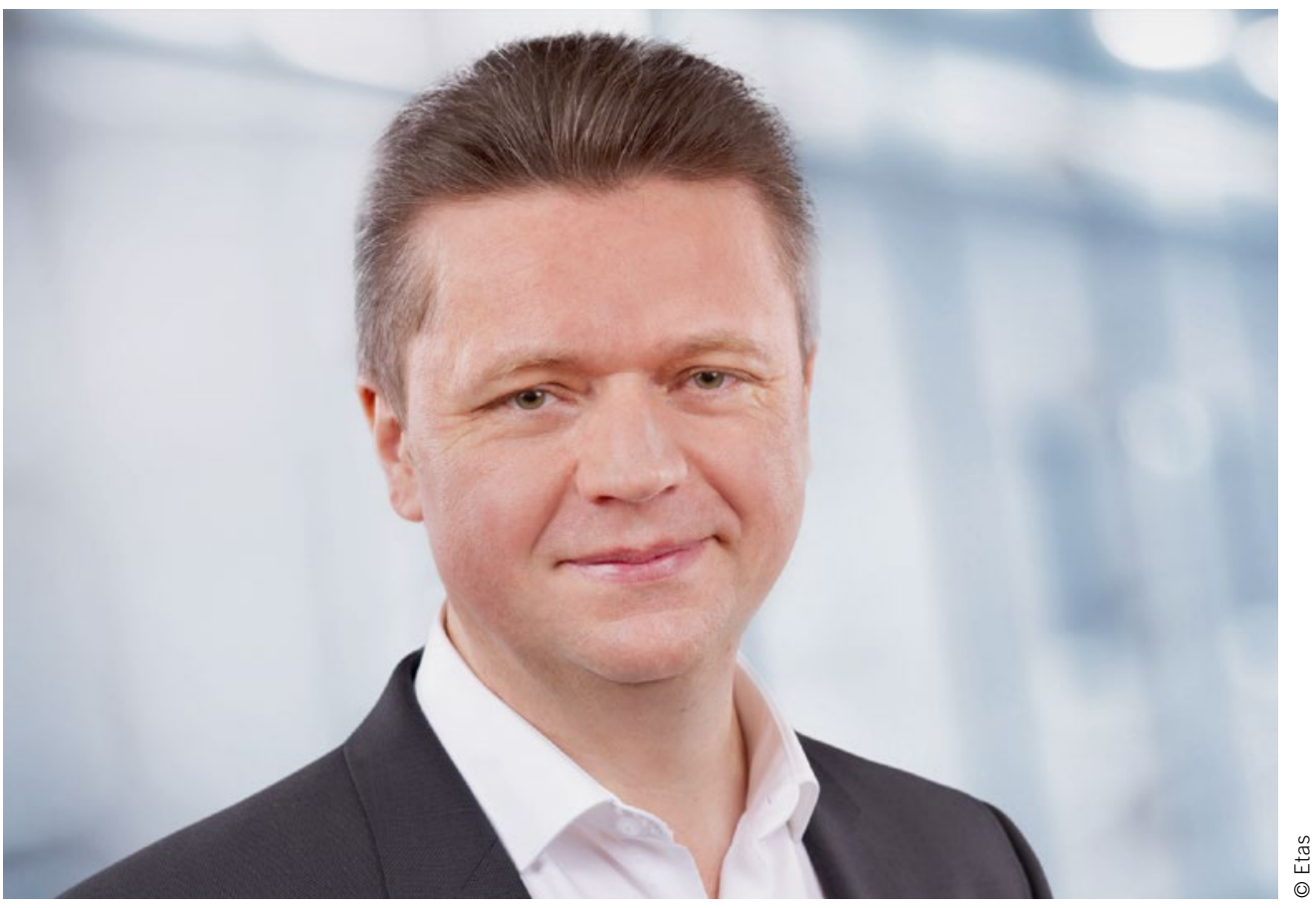

Günter Gromeier

Vice President of the

Automotive Product Group

at Etas

\section{Coordinated Development Solutions are the Key to Success}

Transition, change, transformation, and uncertainty are probably the best words to describe the current situation in the automotive industry. E-mobility, connectivity, (partially) automated driving, mobility services, automotive apps ... The list of technical challenges goes on and on. What will be launched onto the market - and how quickly? However, they all have one thing in common: Software plays the leading role in most innovations.

The pressure to change is enormous. Major players from the world of IT are forcing their way into the market. New mobility business models for vehicles in the field are growing in importance. Many software projects are developed separately from the hardware. In addition, the highest safety and security requirements apply for automotive software - especially in the field of ADAS/AD. Therefore, the challenge is to launch innovative software quickly, safely, and economically onto the market before continuously updating it in the field. Agile development methods, virtual validation, and continuous integration and deployment $(\mathrm{CI} / \mathrm{CD})$ are all prerequisites for success. If we take a closer look at the development process, we can identify four important areas, among others: functional application software, middleware for microcontroller- and microprocessor-based hardware, holistic solutions for cybersecurity, and development tools for the secure and efficient development of complex systems with high data volumes that enable the fastest possible development cycles, whether in a traditional developer workspace or in the cloud. While the application software offers great differentiation potential for the OEMs, middleware, security, and tools have great potential in terms of standardization and common development platforms. Alongside synergy effects, this also offers a higher degree of safety and security. After all, the effort involved in verifying functional safety and in the measures for consistent security increase disproportionately to the software's complexity, degree of connectivity, and update frequency. Only by acting together can this challenge be overcome.

This will enable OEMs and their suppliers to concentrate on the functionality of the application that actually sets it apart as an USP - and reach their objectives quickly. To ensure success, it is imperative that the middleware, security solutions, and development tools are coordinated with one another. This is the only way for development partners to add value to their solutions and achieve their goals. The speed and scope of the changes in automotive software development have never been as significant as they are today. Positive, close cooperation and partnerships are more important than ever for this. The time for pure isolated solutions is definitely over. 